\title{
How transverse thermal fluctuations disorder a condensate of chiral spirals into a quantum spin liquid
}

\author{
Robert D. Pisarski®, ${ }^{1}$ Alexei M. Tsvelik ${ }^{2},{ }^{2}$ and Semeon Valgushev ${ }^{1}$ \\ ${ }^{1}$ Department of Physics, Brookhaven National Laboratory, Upton, New York 11973, USA \\ ${ }^{2}$ Condensed Matter Physics and Materials Science Division, Brookhaven National Laboratory, \\ Upton, New York 11973-5000, USA
}

(Received 21 May 2020; accepted 7 July 2020; published 21 July 2020)

\begin{abstract}
For a scalar theory with a global $O(N)$ symmetry, when $N=2$ a spatially inhomogeneous condensate arises when the term in the Lagrangian with two spatial derivatives has a negative coefficient. If the condensate for such a chiral spiral includes only one mode, characterized by a momentum $k_{0} \hat{z}$, then in perturbation theory at nonzero temperature the propagator for the static mode has a double pole when $\boldsymbol{k}^{2}=k_{0}^{2}$. We conjecture that since chiral spirals spontaneously break both global and spacetime symmetries, that such double poles are a universal property of their static transverse modes. Fluctuations from double poles generate linear infrared divergences in any number of spatial dimensions and disorder the condensate of chiral spirals, analogous to a type of quantum spin liquid. The characteristic feature of this region is that over large spatial distances the two point function is the usual exponential times an oscillatory function. We establish this at large $N$ and suggest that it occurs for all $N>2$. Implications for fermion models and the phase diagram of QCD at nonzero density are discussed.
\end{abstract}

DOI: $10.1103 /$ PhysRevD.102.016015

\section{INTRODUCTION}

Scalar field theories with a global $O(N)$ symmetry are a useful paradigm for the study of phase transitions [1,2]. In vacuum there are two possible phases: symmetric, when the mass squared is positive, and broken, when it is negative. The latter spontaneously breaks the $O(N)$ symmetry and generates Goldstone bosons.

At nonzero temperature or net density Lorentz symmetry is lost, and a different phase can arise. By causality, terms with two time derivatives must always have a positive coefficient. However, for an effective Lagrangian in a medium the coefficient of the term quadratic in the spatial derivatives, $Z$, can be negative. Of course, stability must be ensured by adding terms with four or more spatial derivatives with positive coefficients. When $Z<0$, spatially inhomogeneous condensates arise naturally, as a balance between the negative term with two spatial derivatives against those with higher powers [1-3]. These appear in many systems, from smectic liquid crystals [1,2], to inhomogeneous polymers [4], to complex fluids [5], to chiral spirals in pions and kaons $[3,6-60]$.

Published by the American Physical Society under the terms of the Creative Commons Attribution 4.0 International license. Further distribution of this work must maintain attribution to the author(s) and the published article's title, journal citation, and DOI. Funded by SCOAP ${ }^{3}$.
It is well known that spatially inhomogeneous condensates exhibit a variety of infrared divergences. Due to the anisotropic propagator of a longitudinal phonon mode, they are disordered logarithmically over large distances $[1-3,11-15]$. Fluctuations also ensure that the transition between the symmetric and spatially inhomogeneous phase is of first, and never of second, order [3,61-65]. Lastly, at or below four spatial dimensions infrared divergences prevent the appearance of a Lifshitz point, where the mass squared and $Z$ vanish simultaneously [2-4,66-71].

In the broken phase, the condensate is constant in both the internal and coordinate space, and generates $N-1$ transverse massless modes. When $Z<0$ and $N=2$ the condensate consists of the two fields which rotate into one another as one moves along a fixed direction in space. In this paper we consider how the $N-2$ transverse modes affect spatially inhomogeneous condensates when $N>2$.

In Sec. II we consider a perturbative analysis of the transverse modes. The effective Lagrangian we consider is given in Sec. II A. In Sec. II B we consider the ansatz for a chiral spiral with a single mode, characterized by a momentum along a given direction, $k_{0} \hat{z}$. We show that for a specific choice of Lagrangian, the static mode has a double pole at nonzero momentum, for $\boldsymbol{k}^{2}=k_{0}^{2}$. We generalize this to a wide class of effective Lagrangians in Sec. II C. While our analysis is limited to the ansatz of a single mode for the condensate, we suggest that it is generic. 
Since it occurs at nonzero momentum, such a double pole produces a severe, linear infrared divergence even in the simplest tadpole diagram. This suggests that the theory is in a novel disordered state. This can be established at large $N$, where the theory is soluble by standard techniques $[72,73]$. We give a general discussion of the expansion at large $N$ in Sec. III, and an explicit solution in Sec. IV. This shows that there are only two phases, broken and symmetric. Even so, there are two parts to the symmetric phase: one which is ordinary, and one which we refer to as a type of "quantum spin liquid." While there is no phase transition between the ordinary symmetric phase and the quantum spin liquid, it is easy to distinguish between the two, as the two point function in the ordinary symmetric phase is just the usual exponential(s), while that in the quantum spin liquid is an exponential times an oscillatory function. The quantum spin liquid includes the entire region where mean field theory indicates a condensate of chiral spirals, plus a larger part of the parameter space.

In Sec. $\mathrm{V}$ we conjecture that static, transverse modes produce a quantum spin liquid for all $N>2$. This is in contrast to Kleinert [10], who added a term to the effective Lagrangian which is nonanalytic in the momenta. This generates a double pole and so disorder for all $N \geq 1$ [74], in contradiction to numerous systems in condensed matter which exhibit quasi-long-range order for $N=1$ and 2 [1-5]. Lee, Nakano, Tatsumi, Tsue, and Friman [32] analyzed an $O(4)$ model, but did not find double poles for the static transverse modes [78]. The nature of Goldstone bosons for spatially inhomogeneous condensates has also been studied by Hidaka, Kamikao, Kanazawa, and Noumi [31] in a Nambu-Jona-Lasino (NJL) model [79] and for $N=2$ by Gudnason, Nitta, Sasaki, and Yokokura [75-77].

In Sec. $V$ we also discuss the modifications of the phase diagram from mean field theory [3], and what happens when the $O(N)$ symmetry is only approximate. We show that the linear infrared divergences of the transverse modes only appear at nonzero temperature, while at zero temperature the infrared divergences are only logarithmic [3].

Admittedly, the analogy to a quantum spin liquid in condensed matter systems [2,80-86] is imprecise. Usually, the disorder in a quantum spin liquid arises due to frustration, such as for an antiferromagnet on a triangular lattice in two dimensions. As such, it automatically persists at zero temperature. In contrast, our model exhibits quasilong-range order at zero temperature.

Section VIA proposes a fermion model in $2+1$ dimensions which could be used to test our predictions at nonzero chemical potential. Section VIB briefly considers implications for the phase diagram of quantum chromodynamics (QCD) [87-96].

\section{PERTURBATIVE ANALYSIS}

\section{A. Effective Lagrangian}

Consider the usual form for the effective Lagrangian,

$$
\begin{aligned}
\mathcal{L}_{0}= & \frac{1}{2}\left(\partial_{0} \boldsymbol{\phi}\right)^{2}+\frac{Z}{2}\left(\partial_{i} \boldsymbol{\phi}\right)^{2}-h \boldsymbol{\phi} \cdot \boldsymbol{\phi}_{b}+\frac{1}{2} m^{2} \boldsymbol{\phi}^{2}+\frac{1}{4} \lambda\left(\boldsymbol{\phi}^{2}\right)^{2} \\
& +\frac{1}{6} \kappa\left(\boldsymbol{\phi}^{2}\right)^{3} .
\end{aligned}
$$

Here $\phi$ is an $N$-component vector, and so the theory has a global symmetry of $O(N)$. The background field, $h$, violates the $O(N)$ symmetry; we usually assume it vanishes, and comment briefly what happens when it is nonzero but small. We assume that the theory is applicable in a medium, and so Euclidean invariance need not apply. This allows for the coefficient of the term with two spatial derivatives, $Z$, to differ from that with two time derivatives.

We not only let $Z \neq 1$ in a medium, but allow $Z$ to be negative. In that case, further terms must be added in order to stabilize the theory. These are necessarily nonrenormalizable; we add

$\mathcal{L}_{\mathrm{NR}}=\frac{1}{2 M^{2}}\left(\partial_{i}^{2} \boldsymbol{\phi}\right)^{2}+\frac{1}{2 M_{1}} \boldsymbol{\phi}^{2}\left(\partial_{i} \boldsymbol{\phi}\right)^{2}+\frac{1}{2 M_{2}}\left(\partial_{i} \boldsymbol{\phi}^{2}\right)^{2}$.

The first term, with four spatial derivatives, ensures stability if $M^{2}>0$. The other two terms, with two spatial derivatives and four $\phi$ 's, $\sim 1 / M_{1}$ and $\sim 1 / M_{2}$, can have either sign, but do not play an essential role in our analysis.

We consider a theory in $d+1$ dimensions at a nonzero temperature $T$, so the energy $E_{n}=2 \pi T n, n=0, \pm 1$, $\pm 2 \ldots$. In this case, the most infrared divergent mode is the static mode with zero energy, $E_{n}=0$, and the effective theory is that for the static mode in $d$ dimensions. We comment later in Sec. V how the infrared divergences are less severe at zero temperature. This is obvious, as at zero temperature the integral over a continuous energy, $E$, smooths out the infrared divergences from the spatial momenta of the transverse mode.

In three spatial dimensions, $d=3, \boldsymbol{\phi}$ has dimensions of mass $^{1 / 2}$. The terms in Eq. (2) are all nonrenormalizable, where $M, M_{1}$, and $M_{2}$ are characteristic of some large mass scale generated by the medium. At zero temperature, such as in a medium at nonzero density, $\phi$ has dimensions of mass, and the analogous couplings are $1 / M^{2}, 1 / M_{1}^{2}$, and $1 / M_{2}^{2}$, respectively.

Most of our analysis is not sensitive to the number of spatial dimensions. However, we note that in one spatial dimensions, $d=1, \phi$ is dimensionless. In that case, it is also natural to use a nonlinear Lagrangian, where $\phi^{2}=1$. The nonlinear Lagrangian is 


$$
\begin{aligned}
\mathcal{L}_{2 \operatorname{dim}}= & \frac{1}{2}\left(\partial_{0} \boldsymbol{\phi}\right)^{2}+\frac{Z}{2}\left(\partial_{i} \boldsymbol{\phi}\right)^{2}+\frac{1}{2 M^{2}}\left(\partial^{2} \boldsymbol{\phi}\right)^{2} \\
& +\frac{1}{2 M_{2}^{2}}\left(\left(\partial_{i} \boldsymbol{\phi}\right)^{2}\right)^{2} .
\end{aligned}
$$

Because of the constraint there is no term $\sim 1 / M_{1}$, but there still are two different terms with four spatial derivatives, $\sim 1 / M^{2}$ and $\sim 1 / M_{2}^{2}$.

\section{B. Single mode ansatz}

Henceforth we concentrate on the static mode at nonzero temperature, in zero background field, $h=0$. The condensate we consider arises when two conditions are met: first, a negative mass squared, $m^{2}<0$. In mean field theory, a negative mass term is balanced by a positive term for the quartic coupling (or of higher order, if that is negative). This balancing generates a nonzero expectation value for the field $\langle\boldsymbol{\phi}\rangle=\phi_{0} \hat{\boldsymbol{\phi}}$, for some fixed direction $\hat{\boldsymbol{\phi}}$. For $N>1$, the choice of $\hat{\boldsymbol{\phi}}$ spontaneously breaks the $O(N)$ symmetry. If $N=1$, the only choice is the sign of the condensate, $\langle\phi\rangle= \pm\left|\phi_{0}\right|$, which spontaneously breaks a global Z(2) symmetry.

If the coefficient of the term with two spatial derivatives is negative, $Z<0$, a similar balancing occurs. The theory generates a condensate where $\left\langle\left(\partial_{i} \boldsymbol{\phi}\right)^{2}\right\rangle$ is nonzero, the term in Eq. (2) with four spatial derivatives, $\sim 1 / M^{2}$, stabilizes the theory. If $\partial_{i} \phi \neq 0$, this condensate is spatially inhomogeneous. We always assume that the inhomogeneity is always only in one direction. Condensates in several directions are possible, but typically have higher energy.

The detailed form of the spatially inhomogeneous condensate depends crucially upon $N$. The equation of motion is, from Eqs. (1) and (2),

$$
\begin{aligned}
& \frac{1}{M^{2}}\left(-\partial_{i}^{2}\right)^{2} \boldsymbol{\phi}+\frac{1}{M_{1}}\left(\left(\partial_{i} \boldsymbol{\phi}\right)^{2} \boldsymbol{\phi}-\partial_{i}\left(\boldsymbol{\phi}^{2} \partial_{i} \boldsymbol{\phi}\right)\right) \\
& \quad+\frac{2}{M_{2}} \partial_{i}\left(\left(\partial_{i} \boldsymbol{\phi}^{2}\right) \boldsymbol{\phi}\right)+Z\left(-\partial_{i}^{2}\right) \boldsymbol{\phi} \\
& \quad+\left(m^{2}+\lambda \boldsymbol{\phi}^{2}+\kappa\left(\boldsymbol{\phi}^{2}\right)^{2}\right) \boldsymbol{\phi}=0
\end{aligned}
$$

When $Z$ is positive, the theory generates a constant condensate, $\phi_{0}= \pm \sqrt{-m^{2} / \lambda}$ when $\kappa=0$. When $N=1$, the only way to develop $\left\langle\left(\partial_{i} \boldsymbol{\phi}\right)^{2}\right\rangle \neq 0$ is for the field to oscillate between the vacuum values, $\pm\left|\phi_{0}\right|$, with some periodicity. This "kink" crystal is a solution of the nonlinear differential equation in Eq. (4). For the Gross-Neveu model in $1+1$ dimensions the precise form of the kink crystal can be computed analytically at large $N$, and is not trivial $[18,19,55,56]$. By using non-Abelian bosonization and the truncated conformal spectrum approach, a generalized Gross-Neveu model, with two flavors and three colors, is also exactly soluble in $1+1$ dimensions $[38,43]$.
When $N \geq 2$, however, there is a much simpler ansatz which generates a condensate for both $\phi$ and its spatial derivatives. The magnitude of $\phi$ is kept constant, with the spatial derivative generated by a rotation in the internal space:

$$
\boldsymbol{\phi}_{0}(\boldsymbol{x})=\phi_{0}\left(\cos \left(k_{0} z\right), \sin \left(k_{0} z\right), \mathbf{0}\right)
$$

This is periodic is some fixed direction, $z$, with period $2 \pi / k_{0}$. This choice of the direction $z$ spontaneously breaks the rotation symmetry.

More general solutions are certainly possible. That of Eq. (5) involves only a single mode. The most general solution with this periodicity involves an infinite number of modes:

$$
\boldsymbol{\phi}_{0}(\boldsymbol{x})=\left(\sum_{n=1}^{\infty} c_{n} \cos \left(n k_{0} z\right), \sum_{n=1}^{\infty} s_{n} \sin \left(n k_{0} z\right), \mathbf{0}\right) .
$$

The great advantage of the single mode ansatz of Eq. (5) is that $\boldsymbol{\phi}^{2}$ is a constant, which makes solving the equations of motion trivial. In contrast, for the multimode solution of Eq. (6) $\phi^{2}$ is not constant, and there are an infinite number of parameters which need to be determined, the coefficients $c_{n}$ and $s_{n}$. We only analyze the single mode solution of Eq. (5), assuming that it is the vacuum of our model. Even if a multimode solution has a lower energy classically, we suggest that as for the single mode solution, quantum fluctuations nevertheless disorder the system into a quantum spin liquid.

With the single mode ansatz of Eq. (5), the Lagrangian equals

$$
\begin{aligned}
\mathcal{L}= & \frac{1}{2 M^{2}} k_{0}^{4} \phi_{0}^{2}+\frac{1}{2 M_{1}} k_{0}^{2} \phi_{0}^{4}+\frac{Z}{2} k_{0}^{2} \phi_{0}^{2}+\frac{m^{2}}{2} \phi_{0}^{2} \\
& +\frac{\lambda}{4} \phi_{0}^{4}+\frac{\kappa}{6} \phi_{0}^{6} .
\end{aligned}
$$

We first vary with respect to $k_{0}$,

$$
\frac{\partial}{\partial k_{0}} \mathcal{L}=0
$$

This is equivalent to minimizing the energy per period of the condensate [31]. Because our ansatz has constant $\boldsymbol{\phi}^{2}$, it is independent of $M_{2}$. The solution is

$$
k_{0}^{2}=\left(-Z-\frac{\phi_{0}^{2}}{M_{1}}\right) \frac{M^{2}}{2} .
$$

$k_{0}^{2}$ has to be positive, so this equation can be satisfied if $Z$ is sufficiently large and negative. Under this condition, substituting $k_{0}$ back into the Lagrangian gives 


$$
\begin{aligned}
\mathcal{L}\left(k_{0}, \phi_{0}\right)= & \frac{1}{2}\left(m^{2}-\frac{Z^{2}}{4} M^{2}\right) \phi_{0}^{2}+\frac{1}{4}\left(\lambda-Z \frac{M^{2}}{M_{1}}\right) \phi_{0}^{4} \\
& +\frac{1}{6}\left(\kappa-\frac{3 M^{2}}{4}\right) \phi_{1}^{6} .
\end{aligned}
$$

The equation of motion for the vacuum expectation value is then determined by the solution of

$$
\begin{aligned}
\frac{\partial \mathcal{L}}{\partial \phi_{0}}= & \left(m^{2}-\frac{Z^{2}}{4} M^{2}\right) \phi_{0}+\left(\lambda-Z \frac{M^{2}}{M_{1}}\right) \phi_{0}^{3} \\
& +\left(\kappa-\frac{3}{4} \frac{M_{2}^{2}}{M_{1}^{2}}\right) \phi_{0}^{5}=0 .
\end{aligned}
$$

When a spatially inhomogeneous condensate develops, $Z<0$ and $k_{0} \neq 0$, this affects the couplings of the scalar potential, including both the mass squared, and the quartic and hexatic couplings.

The propagator for the longitudinal modes is involved, and involves a phonon mode associated with the spontaneous breaking of translational symmetry, along $z$, by the condensate [1-3,31,32,75-77].

The propagator for the transverse modes is easy to compute, though

$$
\phi=(\sigma, \chi) .
$$

Then the inverse propagator for a static $\chi$ field is

$$
\begin{aligned}
\Delta_{\chi}^{-1}(E=0, \boldsymbol{k})= & \frac{1}{M^{2}}\left(\boldsymbol{k}^{2}\right)^{2}+\left(Z+\frac{\phi_{0}^{2}}{M_{1}}\right) \boldsymbol{k}^{2} \\
& +\frac{1}{M_{1}} k_{0}^{2} \phi_{0}^{2}+m^{2}+\lambda \phi_{0}^{2}+\kappa \phi_{0}^{4} .
\end{aligned}
$$

Using the expression for $k_{0}$, Eq. (9),

$$
\Delta_{\chi}^{-1}(0, \boldsymbol{k})=\frac{1}{M^{2}}\left(\boldsymbol{k}^{2}-k_{0}^{2}\right)^{2}+\mathcal{M}^{2} .
$$

After some algebra, we find that

$$
\mathcal{M}^{2}=\frac{1}{\phi_{0}} \frac{\partial \mathcal{L}}{\partial \phi_{0}}=0
$$

By direct computation it is not obvious that the mass squared, about $k_{0}$, is proportional to the equation of motion, and so vanishes. We show this for a very general model in the next section, Eq. (21).

That the mass squared vanishes at zero momentum for the transverse modes is simply an expression of Goldstone's theorem. That it does so for a chiral spiral with a single mode, about the characteristic momentum $k_{0}$ of the condensate, is not obvious.

Further, while the transverse modes in Eq. (14) are massless at $k_{0}$, they do so through a double pole, which generates severe infrared divergences. Any tadpole diagram involving the transverse field is proportional to

$$
\int d^{d} \boldsymbol{k} \frac{1}{\left(\boldsymbol{k}^{2}-k_{0}^{2}\right)^{2} / M^{2}+\mathcal{M}^{2}} \sim \frac{M^{2}}{k_{0}^{2}} \int_{k \sim k_{0}} \frac{d k}{\left(k-k_{0}\right)^{2}} .
$$

In any number of dimensions, including $d=1$, this is a linear infrared divergence about $k_{0}$. Consequently, the tadpole diagram blows up, and the ansatz is certainly destabilized once quantum fluctuations are included.

We find a linear infrared divergence from the double pole in the transverse fluctuations again when we analyze the model at large $N$ in Sec. III. The advantage of analyzing the model at large $N$ is that then we can be sure that this infrared divergence is not cut off by other diagrams, or other effects which we might miss in a perturbative analysis.

\section{General Lagrangian for the single mode ansatz}

This result can be generalized to the following Lagrangian:

$$
\mathcal{L}=\frac{1}{2}\left(\partial_{0} \boldsymbol{\phi}\right)^{2}+\sum_{n=1}^{\infty} \frac{Z_{n}}{2} \boldsymbol{\phi}\left(-\partial^{2}\right)^{n} \boldsymbol{\phi}+\frac{v_{n}}{2}\left(\boldsymbol{\phi}^{2}\right)^{n} .
$$

This assumes arbitrary powers of spatial derivatives and of $\phi^{2}$ in the potential. We do assume that terms with spatial derivatives only include two powers of $\phi$, and not higher powers of $\phi$, such as $\boldsymbol{\phi}^{2}\left(\partial_{i} \boldsymbol{\phi}\right)^{2}$ and $\left(\partial_{i} \boldsymbol{\phi}^{2}\right)^{2}$ in Eq. (2). We suspect that such terms could be included, but as the number of spatial derivatives increases so does the number of such terms, and so we simply ignore these to emphasize the physics.

What is essential is that our ansatz involves only a single mode in momentum space, Eq. (5), so that $\boldsymbol{\phi}^{2}$ is of constant magnitude. With this ansatz, for static fields the Lagrangian becomes

$$
\mathcal{L}\left(k_{0}, \phi_{0}\right)=\frac{1}{2} \sum_{n=1}^{\infty} Z_{n} k_{0}^{2 n} \phi_{0}^{2}+v_{n} \phi_{0}^{2 n}
$$

Varying with respect to $\phi_{0}$ gives

$$
\frac{\partial \mathcal{L}}{\partial \phi_{0}}=\sum_{n=1}^{\infty}\left(Z_{n} k_{0}^{2 n}+n v_{n} \phi_{0}^{2 n-2}\right) \phi_{0}=0 .
$$

The solution is either $\phi_{0}=0$ or a nonzero value of $\phi_{0}$. The solutions to Eqs. (8) and (19) are, in general, involved.

However, if the only thing we wish to do is to calculate the transverse propagator, we do not require the explicit form of the solution. This depends crucially upon the point that with a single mode ansatz, $\boldsymbol{\phi}^{2}$ is constant. The inverse propagator for the transverse modes is just 


$$
\Delta^{-1}(\boldsymbol{k})=\sum_{n=1}^{\infty} Z_{n}\left(\boldsymbol{k}^{2}\right)^{n}+n v_{n} \phi_{0}^{2 n-2}
$$

Consider the value of this propagator at a point $\boldsymbol{k}=\hat{k} k_{0}$, where $\hat{k}^{2}=1$ is a unit vector. That is, the momentum must have magnitude $k_{0}$, but need not lie along the $z$ direction of the condensate. Then

$$
\Delta^{-1}\left(k_{0} \hat{k}\right)=\sum_{n=1}^{\infty} Z_{n} k_{0}^{2 n}+n v_{n} \phi_{0}^{2 n-2} .
$$

By the equation of motion for $\boldsymbol{\phi}$, Eq. (19), this vanishes. Thus the transverse modes have a zero at $\boldsymbol{k}=k_{0} \hat{k}$. Next, expand the inverse transverse propagator about this point:

$$
\left.\frac{\partial}{\partial k} \Delta^{-1}(\boldsymbol{k})\right|_{\boldsymbol{k}=\hat{k} k_{0}}=2 \sum_{n=1}^{\infty} n Z_{n}\left(k_{0}\right)^{2 n-1} \hat{k} .
$$

But by the stationary point condition with respect to $k_{0}$, Eq. (8), this also vanishes.

The second derivative of the inverse transverse propagator about $k_{0} \hat{k}$ is

$$
\left.\frac{\partial^{2}}{\partial k^{2}} \Delta^{-1}(\boldsymbol{k})\right|_{\boldsymbol{k}=k_{0} \hat{k}}=2 \sum_{n=1}^{\infty} n(2 n-1) Z_{n}\left(k_{0}\right)^{2 n-2}
$$

There is no reason for this quantity to vanish. Indeed, neither can we be certain that it is positive. However, because of the coefficient $\sim n(2 n-1)$, the terms with the highest $n$ dominate, and it is reasonable to assume so. The positivity of Eq. (23) is, in any case, a necessary condition for stability of the theory.

Since the first derivative of the propagator vanishes at $\hat{k} k_{0}$, and the second does not, this establishes the existence of a double zero, at nonzero momentum, for an extremely general form of the effective Lagrangian.

It is possible that the infrared divergence of the single mode solution is eliminated by going to a multimode solution, Eq. (6). As mentioned previously, we expect that the vacuum with the lowest energy is that with a single mode. Even if this is not true, we suggest that the appearance of double poles in the static, transverse propagator is generic, following from the spontaneous breaking of both the internal and spacetime symmetries. Presumably the double pole is about the smallest periodic momentum, $k_{0}$.

\section{GENERAL ANALYSIS AT LARGE $N$}

\section{A. Positive $Z$}

We treat both symmetric and broken phases simultaneously. For a large $N$ expansion [72,73], we take

$$
\phi=(\sigma, \chi) .
$$

When the symmetry breaks, we assume that $\langle\sigma\rangle \neq 0$. We integrate out the $N-1$ component field $\chi$ by introducing a constraint field,

$$
\mathcal{L}_{\text {cons }}=\frac{i \epsilon}{2}\left(\omega-\sigma^{2}-\chi^{2}\right) .
$$

Integrating out the $\chi$ field, the effective action is

$$
\begin{aligned}
\mathcal{S}= & \int d^{3} x\left(\frac{1}{2 M^{2}}\left(\partial^{2} \sigma\right)^{2}+\frac{Z}{2}\left(\partial_{i} \sigma\right)^{2}+\mathcal{V}(\omega)+\frac{i \epsilon}{2}\left(\omega-\sigma^{2}\right)\right) \\
& +\frac{(N-1)}{2} \operatorname{tr} \log \left(\frac{1}{M^{2}}\left(-\partial^{2}\right)^{2}+Z\left(-\partial^{2}\right)+i \epsilon\right) .
\end{aligned}
$$

Using the constraint, in the potential of Eq. (1) we replace $\phi^{2}$ by $\omega$, to define

$$
\mathcal{V}(\omega)=+\frac{1}{2} m^{2} \omega+\frac{1}{4} \lambda \omega^{2}+\frac{1}{6} \kappa \omega^{3} .
$$

For simplicity we assume that $M_{1}=M_{2}=\infty$ and $h=0$.

Assume that the stationary point for $\sigma$ is constant. Denoting the stationary points by $\sigma_{0}, \epsilon_{0}$, and $\omega_{0}$, the equations of motion give

$$
\epsilon_{0} \sigma_{0}=0
$$

for $\sigma$,

$$
\frac{i}{2} \epsilon_{0}+\left.\frac{\partial}{\partial \omega} \mathcal{V}(\omega)\right|_{\omega=\omega_{0}}=0
$$

for $\omega$, and

$\frac{1}{2}\left(\omega_{0}-\sigma_{0}^{2}\right)+\frac{(N-1)}{2} \operatorname{tr} \frac{1}{\left(-\partial^{2}\right)^{2} / M^{2}+Z\left(-\partial^{2}\right)+i \epsilon_{0}}=0$

for $\epsilon$.

The equation of motion in Eq. (28) is especially useful. In the broken phase $\sigma_{0} \neq 0$, and so $\epsilon_{0}$ vanishes. From Eq. (30), this ensures that the transverse modes are Goldstone bosons. Equation (29) determines $\omega_{0}$, which necessarily has a nonzero value.

Conversely, in the symmetric phase $\sigma_{0}$ vanishes, and so $\epsilon_{0}$ is nonzero, determined by Eq. (29). From Eq. (30), the transverse modes are massive. With some computation, it can be shown that they are degenerate with the longitudinal mode.

$$
\text { B. } Z<\mathbf{0}
$$

We now decompose $\boldsymbol{\phi}$ into $\boldsymbol{\sigma}$ and a $N-2$ component vector, $\chi$, 


$$
\phi=(\sigma, \chi)
$$

We introduce two constraint fields:

$$
\mathcal{L}_{\text {cons }}=\frac{i \epsilon}{2}\left(\omega-\chi^{2}\right)+\frac{i \tilde{\epsilon}}{2}\left(\tilde{\omega}-\left(\partial_{i} \chi\right)^{2}\right) .
$$

Here we only introduce constraint fields for the transverse fluctuations, for both their magnitude and the square of their derivative. This complicates the form of the effective Lagrangian, but ensures that we isolate the dynamics of these modes.

We take $M_{1}=M_{2}=h=\kappa=0$, although again it is not difficult to include them. After integrating out $\chi$, the effective Lagrangian is

$$
\begin{aligned}
\mathcal{S}_{\text {eff }}= & \int d^{3} x\left(\frac{1}{2 M^{2}}\left(\partial^{2} \boldsymbol{\sigma}\right)^{2}+\frac{Z}{2}\left(\partial_{i} \boldsymbol{\sigma}\right)^{2}+\frac{m^{2}}{2} \boldsymbol{\sigma}^{2}+\frac{\lambda}{2} \omega \boldsymbol{\sigma}^{2}\right. \\
& \left.+\frac{\lambda}{4}\left(\boldsymbol{\sigma}^{2}\right)^{2}-\frac{i}{2}(\epsilon \omega+\tilde{\epsilon} \tilde{\omega})+\frac{Z}{2} \tilde{\omega}+\frac{m^{2}}{2} \omega+\frac{\lambda}{4} \omega^{2}\right) \\
& +\frac{(N-2)}{2} \operatorname{tr} \log \left(\frac{1}{M^{2}}\left(-\partial^{2}\right)^{2}-i \partial_{i}\left(\tilde{\epsilon} \partial_{i}\right)+i \epsilon\right) .
\end{aligned}
$$

The expectation value of all quantities are denoted $\epsilon_{0}$, $\omega_{0}$, etc. The equation of motion for $\omega$ is

$$
2 \frac{\partial}{\partial \omega} \mathcal{S}_{\mathrm{eff}}=-i \epsilon_{0}+m^{2}+\lambda\left(\omega_{0}+\boldsymbol{\sigma}_{0}^{2}\right)=0
$$

We introduce the effective mass,

$$
m_{\mathrm{eff}}^{2}=i \epsilon_{0}=m^{2}+\lambda\left(\omega_{0}+\boldsymbol{\sigma}_{0}^{2}\right)
$$

The $\tilde{\omega}$ field only appears in two places, and so its equation of motion,

$$
2 \frac{\partial}{\partial \tilde{\omega}} \mathcal{S}_{\mathrm{eff}}=-i \tilde{\epsilon}_{0}+Z=0
$$

just fixes $i \tilde{\epsilon}_{0}=Z$.

The equation of motion for $\boldsymbol{\sigma}$ is that of Eq. (4),

$$
\frac{\partial}{\partial \boldsymbol{\omega}} \mathcal{S}_{\text {eff }}=\left(\frac{1}{M^{2}}\left(-\partial_{i}^{2}\right)^{2}+Z\left(-\partial^{2}\right)+m_{\mathrm{eff}}^{2}\right) \boldsymbol{\sigma}_{0}=0,
$$

where we use the definition of $m_{\mathrm{eff}}$.

It is useful to introduce the propagator for the static, transverse mode. In momentum space, the static inverse propagator for $\chi$ is, suppressing the isospin indices,

$$
\Delta_{\chi}^{-1}(E=0, \boldsymbol{k})=\frac{1}{M^{2}}\left(\boldsymbol{k}^{2}\right)^{2}+Z \boldsymbol{k}^{2}+m_{\mathrm{eff}}^{2} .
$$

We have used Eq. (34) to fix $\epsilon_{0}$ in terms of $m_{\mathrm{eff}}^{2}$, and Eq. (36) to set $\tilde{\epsilon}_{0}$.

The equation of motion for $\epsilon$ is

$2 i \frac{\partial}{\partial \epsilon} \mathcal{S}_{\mathrm{eff}}=-\omega_{0}+(N-2) \int \frac{d^{3} k}{(2 \pi)^{3}} \Delta_{\chi}(0, \boldsymbol{k})=0$,

while that for $\tilde{\epsilon}$ is

$$
2 i \frac{\partial}{\partial \tilde{\epsilon}} \mathcal{S}_{\text {eff }}=-\tilde{\omega}_{0}+(N-2) \int \frac{d^{3} k}{(2 \pi)^{3}} k^{2} \Delta_{\chi}(0, \boldsymbol{k})=0 .
$$

Remember this is only for the static mode at nonzero temperature, and so a factor of temperature has been absorbed into the couplings and fields. At zero temperature, the corresponding integral is then over all four momenta, $E$ and $\boldsymbol{k}$.

We can now make a straightforward analysis for the ansatz of a chiral spiral with a single mode, Eq. (5). The equation of motion for the $\boldsymbol{\sigma}$ field, Eq. (37), gives

$$
\frac{1}{M^{2}} k_{0}^{4}+Z k_{0}^{2}+m_{\mathrm{eff}}^{2}=0
$$

or $\boldsymbol{\sigma}_{0}=0$. Now it is useful to recognize a remarkable fact, that the inverse propagator in Eq. (38) has exactly the same structure as Eq. (41). Consequently, the (static) inverse transverse propagator vanishes at $k_{0}, \Delta_{\chi}^{-1}\left(0, k_{0} \hat{\boldsymbol{k}}\right)=0$.

This still does not fix the value of $k_{0}$. However, for a physical field the propagator must be positive everywhere or the theory is unstable. It is automatically positive at large $k$, while positivity at $k=0$ implies that $m_{\text {eff }}^{2}>0$. As a quadratic equation in $k_{0}^{2}$, there can be two roots of Eq. (41), at $k_{0}^{-}$and $k_{0}^{+}$. If $k_{0}^{-}<k_{0}^{+}$, the propagator is positive for $k<k_{0}^{-}$, crosses zero at $k_{0}^{-}$, negative when $k_{0}^{-}<k<k_{0}^{+}$, and positive again when $k>k_{0}^{+}$. If $k_{0}^{-} \neq k_{0}^{+}$, then, the propagator is negative for some range of momenta, and the theory is unstable.

The only way that Eq. (41) can be satisfied is if there is only one zero for the inverse propagator, with $k_{0}^{-}=k_{0}^{+}$. This implies that the propagator is extremal at $k_{0}$ :

$$
\left.\frac{\partial}{\partial k} \Delta^{-1}(0, k \hat{\boldsymbol{k}})\right|_{k=k_{0}}=\frac{4}{M^{2}}\left(k_{0}^{2}+\frac{Z}{2} M^{2}\right) k_{0}=0 .
$$

This is the same equation as we obtained by varying the Lagrangian, evaluated for the chiral spiral, with respect to $k_{0}$ in Eq. (9). Since the propagator is extremal at $k_{0}$, the inverse propagator has a double zero at $k_{0}$,

$$
\Delta_{\chi}^{-1}(0, \boldsymbol{k})=\frac{1}{M^{2}}\left(\boldsymbol{k}^{2}-k_{0}^{2}\right)^{2} .
$$

In other words, just by requiring that the theory has a transverse propagator which is everywhere positive, that 
and the equations of motion force a double pole in $\Delta_{\chi}(0, \boldsymbol{k})$ at $k=k_{0}$.

This is precisely the same conclusion as we found perturbatively in Secs. II B and IIC. While before we could only suggest that the tadpole integral over the transverse propagator gives a linear infrared divergence, Eq. (16), at large $N$ these integrals are forced upon us by Eqs. (39) and (40), and there is no escape from a linear infrared divergence.

At large $N$, the $N-2$ fluctuations must be included in order to obtain a self-consistent solution, assuming that the quartic coupling $\lambda N$ is held fixed as $N \rightarrow \infty$. We demonstrate in the next section that there is a self-consistent solution for the theory, as a phase disordered by quantum fluctuations.

\section{EXPLICIT SOLUTION AT LARGE $N$}

In the previous section we showed that assuming a chiral spiral with a single mode produces a double pole in the propagator for the transverse mode. We demonstrate in this section that there is a nonperturbative solution for the symmetric phase, even when the mean field indicates a condensate of chiral spirals, when $m_{\text {eff }}^{2}$ and $Z$ are negative. We start with the Lagrangian of Eqs. (1) and (2), and for simplicity assume $1 / M_{1}=1 / M_{2}=\kappa=0$. It is trivial to generalize our analysis to the general case. Since the constraint field for $\left(\partial_{i} \chi\right)^{2}$ in Eq. (32) did not make a significant difference, we ignore it, and only introduce $\omega=\boldsymbol{\phi}^{2}$, Eq. (25), and integrate over all $\boldsymbol{\phi}$. If the six-point coupling $\kappa$ vanishes, it is possible to integrate over $\omega$ and obtain an effective action only in terms of the constraint field, $\epsilon$ :

$$
\begin{aligned}
\mathcal{S}= & \int d^{3} x \frac{\epsilon^{2}}{4 \lambda} \\
& +\frac{N}{2} \operatorname{tr} \log \left(\frac{1}{M^{2}}\left(-\partial^{2}\right)^{2}+Z\left(-\partial^{2}\right)+m^{2}-i \epsilon\right) .
\end{aligned}
$$

We expand about a saddle point

$$
\epsilon=i \epsilon_{0}+\epsilon_{\mathrm{qu}},
$$

where $\epsilon_{0}$ is determined by

$$
\epsilon_{0}-\frac{\lambda N}{2} \operatorname{tr} \frac{1}{\left(-\partial^{2}\right)^{2} / M^{2}+Z\left(-\partial^{2}\right)+m_{\text {eff }}^{2}}=0,
$$

and we define the renormalized mass,

$$
m_{\mathrm{eff}}^{2}=m^{2}+\epsilon_{0}
$$

We look for the simplest solution, with constant $\epsilon_{0}$. This excludes a chiral spiral condensate which involves multimodes, Eq. (6). As we show at the end of this section, however, our solution is at least locally stable, and so while we cannot exclude it, it appears unlikely that a multimode chiral spiral has a lower action.

For constant $\epsilon_{0}$, the transverse propagator is

$$
\Delta(\boldsymbol{k})=\frac{1}{\left(\boldsymbol{k}^{2}\right)^{2} / M^{2}+Z \boldsymbol{k}^{2}+m_{\mathrm{eff}}^{2}} .
$$

We need to evaluate

$$
\begin{aligned}
\operatorname{tr} \Delta & \equiv \int \frac{d^{3} k}{(2 \pi)^{3}} \Delta(\boldsymbol{k})=\frac{M^{2}}{4 \pi^{2}} \int_{-\infty}^{+\infty} \mathrm{d} k \frac{k^{2}}{\left(k^{2}+m_{+}^{2}\right)\left(k^{2}+m_{-}^{2}\right)} \\
& =\frac{M^{2}}{4 \pi^{2}} \frac{1}{m_{+}^{2}-m_{-}^{2}} \int_{-\infty}^{+\infty} \mathrm{d} k\left(\frac{m_{+}^{2}}{k^{2}+m_{+}^{2}}-\frac{m_{-}^{2}}{k^{2}+m_{-}^{2}}\right),
\end{aligned}
$$

where

$$
m_{ \pm}^{2}=\frac{Z M^{2}}{2}\left(1 \pm \sqrt{1-\alpha^{2}}\right), \quad \alpha=\frac{2 m_{\mathrm{eff}}}{|Z| M} .
$$

To evaluate the integral it is necessary to take care with where the poles lie in the complex $k$ plane.

\section{A. Solution for the effective mass}

We start with the case where $\alpha \leq 1$, so that $m_{ \pm}^{2}$ is real and positive, so we can assume the same for $m_{+}$and $m_{-}$. Equation (49) equals

$$
\operatorname{tr} \Delta=\frac{M^{2}}{4 \pi} \frac{1}{m_{+}+m_{-}} .
$$

As $m_{+}^{2}$ and $m_{-}^{2}$ are solutions to a quadratic equation in $k^{2}$, $\left(k^{2}\right)^{2}+Z M^{2} k^{2}+m_{\mathrm{eff}}^{2} M^{2}=\left(k^{2}+m_{+}^{2}\right)\left(k^{2}+m_{-}^{2}\right)=0$,

$\left(m_{+}+m_{-}\right)^{2}=2 \sqrt{m_{+}^{2} m_{-}^{2}}+m_{+}^{2}+m_{-}^{2}=2 m_{\mathrm{eff}} M+Z M^{2}$.

This is valid for positive $m_{\text {eff }}$ and either sign of $Z$, if the quantity $2 m_{\mathrm{eff}}+Z M$ is positive. The saddle point equation of Eq. (46) becomes

$$
m_{\mathrm{eff}}^{2}-m^{2}=\lambda_{0} \frac{M^{3 / 2}}{\sqrt{2 m_{\mathrm{eff}}+Z M}},
$$

where we define the rescaled quartic coupling,

$$
\lambda_{0}=\frac{\lambda N}{8 \pi} .
$$

At large $N, \lambda_{0}$ and all other quantities, $m_{\text {eff }}, m, M$, and $Z$, are of order one as $N \rightarrow \infty$. The mass dimensions also match, as in three dimensions $\lambda$ has dimensions of mass. 
For given values of $Z, m^{2}$ and $\lambda_{0}$, we need to determine the solution for $m_{\text {eff }}^{2}$. Notice that the left-hand side of Eq. (53) is a monotonically increasing function of $m_{\mathrm{eff}}$, while the right-hand side is a monotonically decreasing function. The solution is then just the intersection of the two. The solution can be found numerically for a given value of the parameters.

It is useful to consider various limits. At large, positive $Z$, the effective mass is

$$
m_{\mathrm{eff}}^{2} \approx m^{2}+\frac{\lambda_{0} M}{\sqrt{Z}}+\cdots, \quad Z \rightarrow+\infty .
$$

Large $Z$ suppresses fluctuations, with the correction to the bare mass $\sim \lambda / \sqrt{Z}$.

As $Z$ decreases, the correction to the effective mass grows. For simplicity, we begin with the line where the bare mass vanishes, $m^{2}=0$. Letting $Z$ decrease, there is a point where $\alpha=2 m_{\text {eff }} /(Z M)=1$; from Eq. (53), this happens when

$$
Z_{1}(0)=\left(2^{3 / 2} \frac{\lambda_{0}}{M}\right)^{2 / 5}, \quad m_{\mathrm{eff}}=\left(\frac{\lambda_{0}^{2} M^{3}}{4}\right)^{1 / 5} .
$$

For arbitrary $m^{2}$, we denote the point at which $\alpha=1$ as $Z_{1}\left(m^{2}\right)$.

For constant $m^{2}=0$, as $Z$ decreases below $Z_{1}(0)$ nothing particularly interesting happens for $m_{\mathrm{eff}}$. For example, when both $Z$ and $m^{2}$ vanish the effective mass remains nonzero,

$$
m_{\mathrm{eff}}=\left(\frac{\lambda_{0}^{2} M^{3}}{2}\right)^{1 / 5}, \quad Z=m^{2}=0 .
$$

Clearly this behavior, $m_{\text {eff }} \sim \lambda_{0}^{2 / 5}$, arises from a nontrivial resummation of perturbation theory at large $N$.

As $Z$ decreases to negative values for $m^{2}=0$, mean field theory suggests that the theory exhibits a condensate of chiral spirals. We demonstrated in the previous section, however, that such a condensate exhibits double poles for the transverse modes, Sec. III B, which disorder the condensate.

However, there is always a self-consistent solution for the symmetric phase, Eq. (53); it is only necessary to ensure that the quantity $2 m_{\text {eff }}+Z M$ is positive. This is easy to do: for example, when $Z$ is large and negative, the solution of Eq. (46) is

$$
m_{\mathrm{eff}}=-Z \frac{M}{2}+\frac{16 \lambda_{0}^{2}}{M} \frac{1}{Z^{4}}+\ldots, \quad Z \rightarrow-\infty .
$$

Thus along the entire line of $m^{2}=0$, for both positive and negative values of $Z$, the theory is in the symmetric phase. This remains valid when $m^{2}$ is positive. The point where $\alpha=1$ changes with $m^{2}$, where $Z_{1}\left(m^{2}\right)$ is a monotonically increasing function of $\mathrm{m}^{2}$.

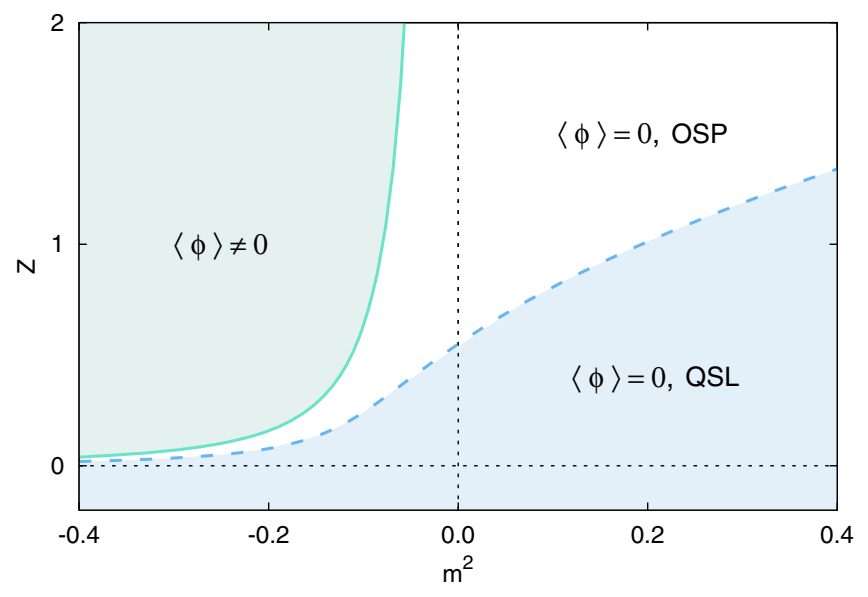

FIG. 1. The phase diagram at large $N$, in the plane of the bare mass squared, $m^{2}$, and the wave function renormalization constant, $Z$, for positive quartic coupling $\lambda=1$. In terms of the global order parameter, there is just the broken phase, where $\langle\phi\rangle \neq 0$, and the symmetric phase, where $\langle\phi\rangle=0$. Nevertheless, there are two parts to the symmetric phase: an ordinary symmetric phase (OSP) and the quantum spin liquid (QSL). In the OSP the two point function of $\phi$ is the usual exponential, Eq. (60), while in the QSL it also oscillates, Eq. (65). The solid line in the upper left quadrant is a line of second order phase transitions. The dashed line in the upper half plane is the boundary between the OSP and the QSL, which is not a phase transition.

When $m^{2}$ becomes negative, the theory enters the broken phase for a fixed, positive value of $Z$. Since the quartic coupling $\lambda$ is positive, this is a second order transition, determined by the condition that the effective mass vanishes. The critical value of the bare mass is given by setting $m_{\text {eff }}^{2}=0$ in Eq. (53), which is just

$$
m_{\text {crit }}^{2}=-\frac{\lambda_{0} M}{\sqrt{Z}}
$$

For $Z>0$ and $m^{2}<-m_{\text {crit }}^{2}$, the theory is in the broken phase. This can be treated following the analysis of Sec. III A, but other than algebraic complications, there are no surprises. The broken phase has massless transverse modes, and a nonzero value for a constant condensate. The phase diagram which results is illustrated in Fig. 1.

In mean field theory, a Lifshitz point occurs when $m^{2}=Z=0$; in the full theory, the corresponding condition is $m_{\text {eff }}^{2}=Z=0$. However, this requires that $m^{2}=m_{\text {crit }}^{2} \rightarrow$ $-\infty$ as $Z \rightarrow 0^{+}$. This is not a Lifshitz point, but a singular limit. That there is no true Lifshitz point agrees with general analysis [2-4,66-71]. We also discuss in the next section why $m_{\text {crit }}^{2} \rightarrow-\infty$ as $Z \rightarrow 0^{+}$.

\section{B. Quantum spin liquid}

The solution for the effective mass obscures interesting physics associated with how the masses $m_{+}$and $m_{-}$change as the bare mass and $Z$ are varied. 
We assume that $m^{2}$ is positive and fixed, and vary $Z$. The extension to negative $m^{2}$ is trivial; it is just necessary to recognize the transition to the broken phase in Fig. 1.

We start at large, positive $Z$, where $\alpha \leq 1$. The masses squared in Eq. (50) are real, so we can take $m_{+}$and $m_{-}$to be real. The poles of the propagator are for $k^{2}=-m_{ \pm}^{2}$, and so along the imaginary axis, at $k= \pm i m_{+}$and $k= \pm i m_{-}$. The two point function of the scalar field is a sum of exponentials,

$$
\left.\left\langle\phi^{i}(x) \phi^{j}(0)\right\rangle\right|_{x \rightarrow \infty}=\delta^{i j}\left(c_{-} \mathrm{e}^{-m_{-} x}+c_{+} \mathrm{e}^{-m_{+} x}\right), \quad \alpha \leq 1 .
$$

At large $Z, m_{-}$is light, $m_{-} \approx m / \sqrt{Z}$, and $m_{+}$heavy, $m_{+} \approx \sqrt{Z} M$. Of course at large distances the light excitation dominates.

As $Z$ decreases from large, positive values, both poles remain on the imaginary axis, as $m_{+}$decreases and $m_{-}$ increases. At the point where $Z=Z_{1}\left(m^{2}\right)$, these poles merge, with

$$
m_{+}=m_{-}=m_{0} \equiv \sqrt{m_{\mathrm{eff}} M} .
$$

When $Z<Z_{1}\left(m^{2}\right)$, the masses squared of $m_{ \pm}^{2}$ have both real and imaginary parts, and the poles of the propagator are at

$$
k=\exp \left( \pm i\left(\frac{\pi}{2} \pm^{\prime} \frac{\theta}{2}\right)\right) m_{0}, \quad Z \geq 0
$$

where

$$
\tan (\theta)=\sqrt{\alpha^{2}-1}, \quad \alpha \geq 1 .
$$

There are four poles, one in each quadrant.

As $Z$ decreases below $Z_{1}\left(m^{2}\right)$, the poles develop both real and imaginary parts. Defining

$m_{r}=m_{0} \cos \left(\frac{\theta}{2}\right), \quad m_{i}=m_{0} \sin \left(\frac{\theta}{2}\right), \quad Z \geq 0$,

and the two point function is

$$
\begin{aligned}
\left.\left\langle\phi^{i}(x) \phi^{j}(0)\right\rangle\right|_{x \rightarrow \infty} & =\delta^{i j} \mathrm{e}^{-m_{r} x}\left(c_{1} \cos \left(m_{i} x\right)+c_{2} \sin \left(m_{i} x\right)\right), \\
\alpha & \geq 1 .
\end{aligned}
$$

Because the poles have a nonzero real part, the two point function is the usual exponential of $m_{r} x$ times cosine or sine of $m_{i} x$. For positive $Z$ less than $Z_{1}\left(m^{2}\right), m_{r}>m_{i}$.

As $Z \rightarrow 0^{+}$, by Eq. (57) the effective mass is nonzero, so $\alpha \rightarrow \infty$ and $\theta=\pi / 2$. The four poles are then at $\pm \pi / 4$ and $\pm 3 \pi / 4$, with $m_{r}=m_{i}=m_{0} / \sqrt{2} ; m_{0}$ is given by Eqs. (57) and (61).
For negative $Z$ the poles are at

$$
\begin{aligned}
& k=\exp \left( \pm \frac{i \theta}{2}\right) m_{0}, \\
& k=\exp \left( \pm i\left(\pi-\frac{\theta}{2}\right)\right) m_{0}, \quad Z \leq 0 .
\end{aligned}
$$

Due to the overall factor of sign of $Z$ in Eq. (50), when $Z$ changes sign the poles rotate by $\pi / 2$. The limit of $Z \rightarrow 0^{ \pm}$ is consistent as then the poles are spaced by $\pi / 2$ anyway.

For negative $Z$ the two point function remains an exponential times oscillatory functions, but now the expressions for $m_{r}$ and $m_{i}$ become

$m_{r}=m_{0} \sin \left(\frac{\theta}{2}\right), \quad m_{i}=m_{0} \cos \left(\frac{\theta}{2}\right), \quad Z \leq 0$,

As $Z$ decreases from zero, $\alpha$ decreases, although it must remain greater than unity. As $Z \rightarrow-\infty$, for example, from Eqs. (58), (61), and (63), $m_{0} \sim|Z|^{1 / 2} M / \sqrt{2}$ and $\theta \sim 8 \lambda_{0} /\left(M|Z|^{5 / 2}\right)$, so that

$m_{r} \approx \frac{2^{3 / 2} \lambda_{0}}{Z^{2}}+\cdots, \quad m_{i} \approx \frac{|Z|^{1 / 2} M}{\sqrt{2}}+\cdots, \quad Z \rightarrow-\infty$.

In mean field theory $m_{\mathrm{eff}}$ vanishes, so the poles of the propagator are at $k^{2}=0$ and $k^{2}=Z M^{2}$. If $Z<0$, the latter is on the axis of real, positive $k^{2}$, with $m_{i}=|Z|^{1 / 2} M$. This indicates the instability of the ground state to the formation of a condensate of chiral spirals. At large $N$, though, instead the theory remains in the symmetric phase. When $Z$ is large and negative, the poles in $k^{2}$ are close to the real axis, with $m_{i} \sim|Z|^{1 / 2} M / \sqrt{2}$, but the phase is stable, as each pole also has a small, real part, $m_{r} \sim \lambda_{0} / Z^{2}$. As $Z$ becomes more negative, the two point function oscillates over distances which are shorter and shorter relative to the distance over which it falls exponentially, $1 / m_{i} \ll 1 / m_{r}$. This is the signal for an unusual form of disorder, which we term a quantum spin liquid [2,80-86].

The boundary between the usual symmetric phase, with the two point function as in Eq. (60), and a quantum spin liquid, with that of Eq. (65), is indicated by a dotted line in Fig. 1. This line is the curve $Z_{1}\left(m^{2}\right)$, where $\alpha=1$. The includes the entire region where $Z<0$, plus a region where $Z$ is small and positive, $0<Z<Z_{1}\left(m^{2}\right)$. This is in contrast to mean field theory, where there is a condensate of chiral spirals only for $Z<0$ and $m^{2}<m_{1}^{2}$, where $m_{1}^{2}>0$; see Fig. 1 of Ref. [3]. This difference is due to quantum fluctuations at large $N$.

While the behavior of the two point function changes as one goes from the ordinary symmetric phase into a quantum spin liquid, in our model this is not a phase 
transition. We have concentrated on the static mode at nonzero temperature. For a static mode with mass squared $m^{2}$, it contributes to the free energy as

$\mathcal{F}(m) \sim T \int d^{3} k\left(\operatorname{tr} \log \left(k^{2}+m^{2}\right)-\frac{m^{2}}{k^{2}}\right) \sim T\left(m^{2}\right)^{3 / 2}$.

In the present case, we have several masses which contribute. For $\alpha \leq 1$ both are real. When $\alpha \geq 1$ there are four complex masses, but they are always paired into complex conjugates, so that the sum is real. Consequently, the free energy, and any finite number of derivatives thereof, behave smoothly as $\alpha \rightarrow 1$.

Logically, it is possible that the imaginary part of the mass, $m_{i}$, vanishes at the same time as the real part, $m_{r}$; if so, this might produce a novel critical point. From the phase diagram of Fig. 1, though, this does not occur: the dotted line, separating the ordinary symmetric phase and the quantum spin liquid, never intersects the line of second order phase transitions.

This also helps to understand one feature of the phase diagram, which is that the bare mass squared diverges, $m_{\text {crit }}^{2} \rightarrow-1 / \sqrt{Z}$, as $Z \rightarrow 0$. This occurs because the broken phase cannot exist for negative $Z$, so the transition to the symmetric phase arises for a small but nonzero value of $Z$.

\section{Stability of the quantum spin liquid phase}

While we have established that there is a novel solution for the symmetric phase when $\alpha>1$, we also need to show that it is stable. This is actually direct. From the expansion of $\epsilon$ in Eq. (45), the two point function of $\epsilon_{\mathrm{qu}}$ is, in momentum space,

$\left\langle\epsilon_{\mathrm{qu}}(\boldsymbol{p}) \epsilon_{\mathrm{qu}}(-\boldsymbol{p})\right\rangle=+\frac{1}{2 \lambda}+N \int \frac{\mathrm{d}^{3} k}{(2 \pi)^{3}} \Delta(\boldsymbol{k}) \Delta(\boldsymbol{k}+\boldsymbol{p})$,

where $\Delta$ is the transverse propagator of Eq. (48). Notice that the sign of the second term is positive in Eq. (70) because the contour of integration for $\epsilon_{\mathrm{qu}}$ runs along the real axis. This is standard in a large $N$ expansion [72,73].

It is also direct to compute the propagator for the $\tilde{\epsilon}$ field of Eq. (33):

$$
\begin{aligned}
& \left\langle\tilde{\epsilon}_{\mathrm{qu}}(\boldsymbol{p}) \tilde{\epsilon}_{\mathrm{qu}}(-\boldsymbol{p})\right\rangle \\
& =+\frac{1}{2 \lambda}+4 N \int \frac{\mathrm{d}^{3} k}{(2 \pi)^{3}}(\boldsymbol{k} \cdot(\boldsymbol{k}+\boldsymbol{p}))^{2} \Delta(\boldsymbol{k}) \Delta(\boldsymbol{k}+\boldsymbol{p}) .
\end{aligned}
$$

Because of the complicated pole structure of the propagators, it is involved to explicitly evaluate these inverse propagators. However, both Eqs. (70) and (71) are convergent in both the ultraviolet and infrared limits. Since the propagator $\Delta \sim 1 /\left(\boldsymbol{k}^{2}\right)^{2}$ at large momentum, ultraviolet convergence follows directly by power counting. Infrared convergence is guaranteed because the inverse propagator for our solution is always gapped. Thus both propagators are convergent integrals over positive quantities, and so are also positive. This implies local stability.

This is important because when $Z<0$, it is possible that at large $N$ there is a multimode solution for a chiral spiral, Eq. (6). We expect that such a solution has a double pole at $k_{0}$, but cannot prove this. However, the quantum spin liquid solution is at least locally stable, and it seems very likely that it is the global minimum.

\section{PHASE DIAGRAM INCLUDING TRANSVERSE FLUCTUATIONS}

In mean field theory, there are three phases: symmetric, broken, and one with a spatially anisotropic condensate [3]. For now we allow $N$ to be arbitrary, so the latter can be either a kink crystal, for $N=1$, or a chiral spiral, for $N \geq 2$. The transition between the symmetric and broken phases is of second order (when the quartic coupling $\lambda>0$ ); that between the symmetric and chiral spiral phases is of second order; and that between the broken and chiral spiral phase is first order, Fig. 1 of Ref. [3].

Fluctuations [61-65] turn the transition between the symmetric and chiral spiral phases into a line of first order transitions, Fig. 2 of Ref. [3]. This is due to fluctuations in the longitudinal mode, whose propagator is

$$
\Delta_{\text {long }}(\boldsymbol{k}) \sim \frac{1}{\delta k_{z}^{2}+\left(4 k_{0} \delta k_{z}+\tilde{k}^{2}\right) \tilde{k}^{2} / M^{2}+m^{2}} .
$$

Here we assume that the condensate is along the $z$ direction, $k_{0}$ the characteristic momentum of the condensate, $\delta k_{z}=$ $k_{z}-k_{0}$ and $\tilde{k}^{2}=\left(\delta k_{z}\right)^{2}+k_{\perp}^{2}$. Notably, there are no terms quadratic in $k_{\perp}$. In mean field theory, the transition between the symmetric and spatially anisotropic condensate phase occurs when $m^{2}=0$. As pointed out by Brazovski $[3,61-65]$, this gives rise to a linear infrared divergence. The transition occurs as the parameters $m^{2}$ and $Z$ are changed, so $m^{2}$ jumps from one nonzero value to another, through a first order transition.

This is very similar to the mechanism proposed in this paper, by which (static) transverse fluctuations disorder a chiral spiral when $N \geq 2$. We find that the would be Goldstone bosons have a double pole when $k=k_{0}$, although not along the direction of the condensate. This produces a tadpole diagram which is linearly divergent in the infrared, Eq. (16). This occurs throughout the chiral spiral phase, however. As we demonstrated in the previous section, Sec. IV, at large $N$ the transverse fluctuations disorder the chiral spiral phase, with a propagator which is

$$
\Delta^{i j}(E, \boldsymbol{k})=\frac{\delta^{i j}}{E^{2}+\left(\boldsymbol{k}^{2}\right)^{2} / M^{2}+Z \boldsymbol{k}^{2}+m_{\mathrm{eff}}^{2}} .
$$


The isospin indices are $i$ and $j$, so the propagator is symmetric. At large $N$ we showed that even in a region where one expects a condensate of chiral spirals-when $m^{2}$ and $Z$ are both negative - that at nonzero temperature static transverse fluctuations disorder the system.

At large $N$ we find that there is no phase transition between the ordinary symmetric phase and that with a quantum spin liquid, only between the ordinary symmetric phase and the broken phase, Fig. 1.

While our conclusions are only certain at large $N$, we suggest that they hold for any $N \geq 2$. Consider the effective Lagrangian of Eq. (33), and consider an expansion about $N=2+\delta$, where $\delta \ll 1$. In this instance, we can expand in $\delta$. The leading terms are given by the classical theory, and quantum fluctuations about that. However, the transverse terms, $\sim \delta$, inevitably bring in the infrared divergences of the would be Goldstone modes, and will disorder the system.

This does not exclude the possibility that the theory is disordered for $\delta \ll 1$, ordered for some intermediate range of $N$, from $N=3$ to $N_{\max }$, and then disordered again for $N>N_{\max }$. This can be studied most directly through numerical simulations of the scalar theory. One of us has performed numerical simulations for $N=8$ and 10 which indicate there is no phase with a condensate of chiral spirals [97]. In Sec. VI A, we suggest a NambuJona-Lasino model, where $N=3$, where this could also be analyzed at nonzero chemical potential.

These numerical simulations [97] show that even if there is a $N_{\max }$, it is difficult to distinguish between a standard symmetric phase and a condensate of chiral spirals. If there is a line of first order transitions between the two phases, as predicted by the analysis of Brazovski [3,61-65], that simplifies things greatly.

In all of our analyses we have assumed that the $O(N)$ symmetry is exact. If this symmetry is broken by a small but nonzero background field, $h \neq 0$ in Eq. (1), even for small $h$, it is not trivial solving for the explicit form of the chiral spiral. The assumption which simplified the analysis so greatly, that $\boldsymbol{\phi}^{2}$ is constant, no longer holds. It is then like the case of a kink crystal, where it is necessary to solve a nonlinear differential equation to determine the form of how a deformed chiral spiral, for small $h$, goes over to a kink crystal, for large $h$.

Even so, for small $h$ it is very natural to assume that the form of the propagator is like that of Eq. (73): for either sign of $Z$, the propagator is always gapped, with $m_{\text {eff }}^{2} \neq 0$. Thus for small $h$, it is reasonable to expect that the quantum spin liquid exists for a large range of parameter space.

We have concentrated on the static mode at nonzero temperature. At zero temperature, the propagator for the transverse mode about a chiral spiral, for the tadpole diagram analogous to Eq. (16),

$$
\begin{aligned}
& \sim \int_{\infty}^{+\infty} \frac{d E}{2 \pi} \int \frac{d^{3} k}{(2 \pi)^{3}} \frac{1}{E^{2}+\left(\boldsymbol{k}^{2}-k_{0}^{2}\right)^{2} / M^{2}} \\
& \sim \int \frac{d^{3} k}{(2 \pi)^{3}} \frac{M}{\left|\boldsymbol{k}^{2}-k_{0}^{2}\right|},
\end{aligned}
$$

after integrating over the energy, $E$, which is continuous at zero temperature. This has a logarithmic infrared divergence about $k=k_{0}$, which mildly washes out a chiral spiral condensate. This disorder is precisely analogous to that which is expected for the phonon of the longitudinal mode [1-3,10-15].

This implies that at sufficiently low temperature, that the phase diagram is like that of mean field theory: there is quasi-long-range order in a chiral spiral phase, separated by a line of first order transitions between that and the symmetric or ordered phases $[3,61-65]$. In the two dimensional space of temperature and whatever thermodynamic variable corresponds to $m^{2}$ and/or $Z$, there are then four phases: the usual broken and symmetric, a quantum spin liquid at nonzero temperature, and a chiral spiral phase at low temperature. While such a structure is surely involved, it does suggest that when studying effective models, it is imperative to consider not only which structure minimizes the free energy in mean field theory, but to include the effects of fluctuations, at least to one loop order.

\section{IMPLICATIONS}

We have established that static transverse modes disorder a condensate of chiral spirals with a single mode. Our analysis can be tested by numerical simulations of the scalar field theory. It is of interest to study these effects for fermions at nonzero density. We discuss two examples.

\section{A. Four fermion models}

In $1+1$ dimensions, Gross-Neveu models are given by

$$
\mathcal{L}=\bar{\psi} \not \partial \psi+g_{1}(\bar{\psi} \psi)^{2}+g_{2}\left(\bar{\psi} \gamma_{5} \psi\right)^{2} .
$$

We take $N_{f}$ flavors of two component fermions, with an implied sum over flavors: $\bar{\psi} \psi \equiv \sum_{i=1}^{N_{f}} \overline{\psi^{i}} \psi^{i}$, etc. The theory is asymptotically free and soluble at large [98] and indeed any $[38,43] N_{f}$. When $g_{2}=0$, the generation of mass, $\langle\bar{\psi} \psi\rangle \neq 0$, spontaneously breaks a $Z(2)$ symmetry. For the chiral Gross-Neveu model, $g_{1}=g_{2}$, mass generation spontaneously breaks the global $U(1)$ symmetry of $\psi \rightarrow \exp \left(i \theta \gamma_{5}\right)$. The global symmetry is larger than $U\left(N_{f}\right)$, equal to $O\left(2 N_{f}\right)$ [99], but this symmetry is respected by the dynamical generation of mass.

These theories are soluble at nonzero chemical potential. At large $N_{f}$ the Gross-Neveu model develops a kink crystal $[18,19,55,56]$. In the chiral Gross-Neveu model at large $N_{f}$ [20-22], the critical temperature $T_{c}$ is independent of $\mu$ : 
when $T=T_{c}$ this has a single mode, but for $T<T_{c}$, it is a twisted kink, which in our terminology is multimode, Eq. (6); see the discussion in Fig. 4 of Ref. [20]. Numerical simulations of Gross-Neveu models have also been carried out at small $N_{f}$, and support the phase diagram found at large $N_{f}$ [57-60].

We note that a type of Gross-Neveu model in $1+1$ dimensions, with two flavors and three colors, is soluble in the limit of zero bare quark mass, and at small mass by using a truncated conformal spectrum approach $[38,43]$. In the chiral limit, only a discrete $Z(2)$ symmetry is spontaneously broken. There is a wealth of phases with quasilong-range order at nonzero chemical potential, including the Bose condensation of either scalar mesons or deuterons, and a phase with gapless baryons.

Four fermion theories are nonrenormalizable in $2+1$ dimensions, but they can still be considered as a type of effective theory. Simulations with the Gross-Neveu model in $2+1$ dimensions [60] find a kink crystal, but one which appears to vanish as the lattice spacing goes to zero.

We cannot use a Gross-Neveu model to study our effect, since the global symmetry is not broken. Indeed, since Goldstone bosons have logarithmic infrared divergences in two spacetime dimensions, it is probably more useful to study models in $2+1$ dimensions.

We suggest the following theory. Let $\psi$ and $\chi$ represent two component fermions in $2+1$ dimensions, with again an implicit sum over $N_{f}$ flavors, $\bar{\psi} \psi=\sum_{i=1}^{N_{f}} \overline{\psi^{i}} \psi^{i}$, and similarly for $\chi$. Combine them together as $\zeta^{i}=\left(\psi^{i}, \chi^{i}\right)$, with the Lagrangian

$$
\mathcal{L}=\bar{\zeta} \not \partial \zeta+g_{1}(\bar{\zeta} \zeta)^{2}+g_{0}\left(\bar{\zeta} \sigma^{a} \zeta\right)^{2},
$$

where the Pauli matrix $\sigma^{a}$ acts in the space of $(\psi, \chi)$. In three spacetime dimensions the mass for a two component fermion is odd under parity and time reversal. A parity even mass is formed by pairing up $\psi$ and $\chi$ together, with masses of equal magnitude and opposite sign [100].

If $g_{0}=0$, the coupling $g_{1}$ may spontaneously break the $Z(2)$ symmetry, but not the $U\left(2 N_{f}\right)$ symmetry, and so a dynamically generated mass is odd under parity. In contrast, if $g_{0}=0$ and $g_{1} \neq 0$, it is very possible that the theory spontaneously generates a parity even mass. In particular, the term $\bar{\zeta} \sigma^{3} \zeta$ tends to give masses of opposite sign to $\psi$ and $\chi$, and breaks $U\left(2 N_{f}\right)$ to $U\left(N_{f}\right) \times U\left(N_{f}\right)$ [100]. For a single flavor $S U(2)$ breaks to $U(1)$, times an overall, unbroken $U(1)$. One of the $S U(2)$ directions generates a condensate, leaving two Goldstone bosons. At nonzero density one of the Goldstone bosons can pair with the condensate to form a chiral spiral, leaving one Goldstone boson to disorder the condensate of chiral spirals.

This model is presumably soluble at large $N_{f}$. We note, however, that the transverse fluctuations are of $\sim N_{f}^{0}$, and so if a chiral spiral condensate arises at infinite $N_{f}$, it is only disordered at next to leading order in $1 / N_{f}$. It may be more useful to use numerical simulations on the lattice, especially for $N_{f}=1$. This assumes that the physics does not disappear as the cutoff vanishes [60]. However, it is easy to add additional, dynamical scalar fields and construct a model which is both renormalizable and with the same pattern of symmetry breaking, Sec. 4.4 of [73].

As discussed in Sec. II B, though, we expect that the spontaneous breaking of an $O(4)$ symmetry prefers a chiral spiral over a kink crystal.

\section{B. Phase diagram of QCD}

Our analysis is relevant for the phase diagram of QCD, in the plane of temperature, $T$, and the quark chemical potential, $\mu$. At nonzero chemical potential, it is natural that the effect of fermion loops turns both the quartic coupling, $\lambda$, and $Z$ negative, Eq. (1) $[3,6-17,23-54]$. In particular, when $\lambda$ changes sign, a critical end point can arise [87-96].

It is not clear what the relationship is between the critical end point and the region where a chiral spiral arises. In the simplest Nambu-Jona-Lasino models, the critical end point coincides with the Lifshitz point (where $Z=m^{2}=0$ ) [29], but in general the two are separate [30]. In any case, fluctuations wash out the Lifshitz point [2-4,66-71]. Calculations using the functional renormalization group appear to show that the region where $Z<0$ is large, while the critical region for the end point is small [95].

In $\mathrm{QCD}$, the order parameter for a chiral spiral only involves the global $U(1)$ symmetry [3]. This is not directly affected by our analysis, as a chiral spiral for $U(1)$ has no transverse modes. Nevertheless, there is surely a close relation between the full chiral symmetry, which is at least an approximate $O(4)$ symmetry, and this $U(1)$. In particular, the $U(1)$ symmetry can exhibit a chiral spiral at any temperature, while as we discussed at the end of Sec. V, an $O(4)$ chiral spiral only exhibits quasi-long-range order at low temperature. The relationship between the two types of symmetry breaking in the phase diagram of QCD is surely intricate, and left to future study.

Even so, it is reasonable to conjecture that any region where $Z<0$, and $U(1)$ chiral spirals arise, affects the propagation of pions and kaons. We do not expect that the inverse propagator of pions or kaons vanishes at any point, as that would produce double poles. It is natural to conjecture that the dispersion relation of pions and kaons is like that of the symmetric modes in Eq. (73).

This modest assumption has immediate implications. If the Minkowski energy $\tilde{E}=i E$, the poles of the propagator in Eq. (73) are

$$
\tilde{E}(\boldsymbol{k})^{2}=\frac{\left(\boldsymbol{k}^{2}\right)^{2}}{M^{2}}+Z \boldsymbol{k}^{2}+m_{\mathrm{eff}}^{2} .
$$

Using the standard Bose-Einstein statistical distribution function, such a modified dispersion relation produces what 
appears to be deviations from a thermal distribution. In this case, the system is thermal, but the dispersion relation is modified. The effects of a modified dispersion relation could be quite striking [101]. This is diluted by integrating over the temperature history and large boost velocity of the medium. It is surely necessary not to look at total abundances, integrated over all momentum, but as a function of momentum. A more sensitive probe is to measure the fluctuations for particles binned with respect to their momentum.

\section{ACKNOWLEDGMENTS}

R. D. P., A. M. T., and S.V. thank the U.S. Department of Energy for support under Contract No. DESC0012704. We thank Y. Hidaka for collaborating at the beginning of this project, and he and E. Nakano for discussions. We thank B. Friman for an extensive correspondence, especially for pointing out the work of Kleinert [10] to us.
[1] P. M. Chaikin and T. C. Lubensky, Principles of Condensed Matter Physics (Cambridge University Press, Cambridge, 2010).

[2] E. H. Fradkin, Field Theories of Condensed Matter Physics (Cambridge University Press, Cambridge, England, 2013), Vol. 82.

[3] R. D. Pisarski, V. V. Skokov, and A. M. Tsvelik, Fluctuations in cool quark matter and the phase diagram of quantum chromodynamics, Phys. Rev. D 99, 074025 (2019).

[4] G. H. Fredrickson, The Equilibrium Theory of Inhomogeneous Polymers (Clarendon Press, Oxford, 2010).

[5] M.E. Cates, Complex fluids: The physics of emulsions, arXiv:1209.2290.

[6] A. W. Overhauser, Structure of Nuclear Matter, Phys. Rev. Lett. 4, 415 (1960).

[7] A. B. Migdal, Pion condensation, Zh. Eksp. Teor. Fiz. 61, 2209 (1971) [Sov. Phys. JETP 34, 1184 (1972)].

[8] A. B. Migdal, Pi Condensation in Nuclear Matter, Phys. Rev. Lett. 31, 257 (1973).

[9] A. B. Migdal, Pion fields in nuclear matter, Rev. Mod. Phys. 50, 107 (1978).

[10] H. Kleinert, No pion condensate in nuclear matter due to fluctuations, Phys. Lett. 102B, 1 (1981).

[11] K. Kolehmainen and G. Baym, Pion condensation at finite temperature. Simple models including thermal excitations of the pion field, Nucl. Phys. A382, 528 (1982).

[12] G. Baym, B. L. Friman, and G. Grinstein, Fluctuations and long range order in finite temperature pion condensates, Nucl. Phys. B210, 193 (1982).

[13] G. G. Bunatian and I. N. Mishustin, Thermodynamical theory of pion condensation, Nucl. Phys. A404, 525 (1983).

[14] T. Takatsuka and R. Tamagaki, $\pi^{0}$ condensation in dense symmetric nuclear matter at finite temperature, Prog. Theor. Phys. 77, 362 (1987).

[15] A. B. Migdal, E. E. Saperstein, M. A. Troitsky, and D. N. Voskresensky, Pion degrees of freedom in nuclear matter, Phys. Rep. 192, 179 (1990).

[16] D. B. Kaplan and A. E. Nelson, Strange goings on in dense nucleonic matter, Phys. Lett. B 175, 57 (1986).

[17] G. E. Brown, C.-H. Lee, M. Rho, and V. Thorsson, From kaon-nuclear interactions to kaon condensation, Nucl. Phys. A567, 937 (1994).
[18] S.-S. Shei, Semiclassical bound states in a model with chiral symmetry, Phys. Rev. D 14, 535 (1976).

[19] M. Thies, From relativistic quantum fields to condensed matter and back again: Updating the Gross-Neveu phase diagram, J. Phys. A 39, 12707 (2006).

[20] G. Basar and G. V. Dunne, Self-Consistent Crystalline Condensate in Chiral Gross-Neveu and Bogoliubovde Gennes Systems, Phys. Rev. Lett. 100, 200404 (2008).

[21] G. Basar and G. V. Dunne, A twisted kink crystal in the chiral Gross-Neveu model, Phys. Rev. D 78, 065022 (2008).

[22] G. Basar, G. V. Dunne, and M. Thies, Inhomogeneous condensates in the thermodynamics of the chiral NJL(2) model, Phys. Rev. D 79, 105012 (2009).

[23] T. Kojo, Y. Hidaka, L. McLerran, and R. D. Pisarski, Quarkyonic chiral spirals, Nucl. Phys. A843, 37 (2010).

[24] T. Kojo, R. D. Pisarski, and A. M. Tsvelik, Covering the Fermi surface with patches of quarkyonic chiral spirals, Phys. Rev. D 82, 074015 (2010).

[25] T. Kojo, Y. Hidaka, K. Fukushima, L. D. McLerran, and R. D. Pisarski, Interweaving chiral spirals, Nucl. Phys. A875, 94 (2012).

[26] T. Kojo, Chiral spirals from noncontinuous chiral symmetry: The Gross-Neveu model results, Phys. Rev. D 90, 065030 (2014).

[27] D. Nickel, How Many Phases Meet at the Chiral Critical Point?, Phys. Rev. Lett. 103, 072301 (2009).

[28] D. Nickel, Inhomogeneous phases in the Nambu-JonaLasino and quark-meson model, Phys. Rev. D 80, 074025 (2009).

[29] M. Buballa and S. Carignano, Inhomogeneous chiral condensates, Prog. Part. Nucl. Phys. 81, 39 (2015).

[30] S. Carignano, M. Buballa, and B.-J. Schaefer, Inhomogeneous phases in the quark-meson model with vacuum fluctuations, Phys. Rev. D 90, 014033 (2014).

[31] Y. Hidaka, K. Kamikado, T. Kanazawa, and T. Noumi, Phonons, pions and quasi-long-range order in spatially modulated chiral condensates, Phys. Rev. D 92, 034003 (2015).

[32] T.-G. Lee, E. Nakano, Y. Tsue, T. Tatsumi, and B. Friman, Landau-Peierls instability in a Fulde-Ferrell type inhomogeneous chiral condensed phase, Phys. Rev. D 92, 034024 (2015). 
[33] M. Buballa and S. Carignano, Inhomogeneous chiral symmetry breaking in dense neutron-star matter, Eur. Phys. J. A 52, 57 (2016).

[34] J. Braun, F. Karbstein, S. Rechenberger, and D. Roscher, Crystalline ground states in Polyakov-loop extended Nambu-Jona-Lasinio models, Phys. Rev. D 93, 014032 (2016).

[35] S. Carignano, E. J. Ferrer, V. de la Incera, and L. Paulucci, Crystalline chiral condensates as a component of compact stars, Phys. Rev. D 92, 105018 (2015).

[36] A. Heinz, F. Giacosa, M. Wagner, and D. H. Rischke, Inhomogeneous condensation in effective models for QCD using the finite-mode approach, Phys. Rev. D 93, 014007 (2016).

[37] S. Carignano, M. Buballa, and W. Elkamhawy, Consistent parameter fixing in the quark-meson model with vacuum fluctuations, Phys. Rev. D 94, 034023 (2016).

[38] P. Azaria, R. M. Konik, Ph. Lecheminant, T. Palmai, G. Takacs, and A. M. Tsvelik, Particle formation and ordering in strongly correlated fermionic systems: Solving a model of quantum chromodynamics, Phys. Rev. D 94, 045003 (2016).

[39] P. Adhikari and J. O. Andersen, Consistent regularization and renormalization in models with inhomogeneous phases, Phys. Rev. D 95, 036009 (2017).

[40] P. Adhikari and J. O. Andersen, Chiral density wave versus pion condensation in the $1+1$ dimensional NJL model, Phys. Rev. D 95, 054020 (2017).

[41] J. O. Andersen and P. Kneschke, Inhomogeneous phases at finite density in an external magnetic field, arXiv:1710 .08341 .

[42] P. Adhikari, J. O. Andersen, and P. Kneschke, Inhomogeneous chiral condensate in the quark-meson model, Phys. Rev. D 96, 016013 (2017).

[43] A. J. A. James, R. M. Konik, P. Lecheminant, N. J. Robinson, and A. M. Tsvelik, Nonperturbative methodologies for low-dimensional strongly correlated systems: From non-Abelian bosonization to truncated spectrum methods, Rep. Prog. Phys. 81, 046002 (2018).

[44] S. Carignano, L. Lepori, A. Mammarella, M. Mannarelli, and G. Pagliaroli, Scrutinizing the pion condensed phase, Eur. Phys. J. A 53, 35 (2017).

[45] T. G. Khunjua, K. G. Klimenko, R. N. Zhokhov, and V. C. Zhukovsky, Inhomogeneous charged pion condensation in chiral asymmetric dense quark matter in the framework of $\mathrm{NJL}_{2}$ model, Phys. Rev. D 95, 105010 (2017).

[46] T. G. Khunjua, K. G. Klimenko, and R. N. Zhokhov, Dense baryon matter with isospin and chiral imbalance in the framework of $\mathrm{NJL}_{4}$ model at large $N_{c}$ : Duality between chiral symmetry breaking and charged pion condensation, Phys. Rev. D 97, 054036 (2018).

[47] J. O. Andersen and P. Kneschke, Chiral density wave versus pion condensation at finite density and zero temperature, Phys. Rev. D 97, 076005 (2018).

[48] S. Carignano, M. Schramm, and M. Buballa, Influence of vector interactions on the favored shape of inhomogeneous chiral condensates, Phys. Rev. D 98, 014033 (2018).
[49] M. Buballa and S. Carignano, Inhomogeneous chiral phases away from the chiral limit, Phys. Lett. B 791, 361 (2019).

[50] T. G. Khunjua, K. G. Klimenko, and R. N. Zhokhov, Dualities in dense quark matter with isospin, chiral, and chiral isospin imbalance in the framework of the large- $\mathrm{N}_{c}$ limit of the $\mathrm{NJL}_{4}$ model, Phys. Rev. D 98, 054030 (2018).

[51] T. G. Khunjua, K. G. Klimenko, and R. N. Zhokhov, Chiral imbalanced hot and dense quark matter: NJL analysis at the physical point and comparison with lattice QCD, Eur. Phys. J. C 79, 151 (2019).

[52] S. Carignano and M. Buballa, Inhomogeneous chiral condensates in three-flavor quark matter, Phys. Rev. D 101, 014026 (2020).

[53] T. G. Khunjua, K. G. Klimenko, and R. N. Zhokhov, Dualities and inhomogeneous phases in dense quark matter with chiral and isospin imbalances in the framework of effective model, J. High Energy Phys. 06 (2019) 006.

[54] T. G. Khunjua, K. G. Klimenko, and R. N. Zhokhov, Charged pion condensation and duality in dense and hot chirally and isospin asymmetric quark matter in the framework of the $\mathrm{NJL}_{2}$ model, Phys. Rev. D 100, 034009 (2019).

[55] M. Thies, Phase structure of the $(1+1)$-dimensional Nambu-Jona-Lasinio model with isospin, Phys. Rev. D 101, 014010 (2020).

[56] M. Thies, First-order phase boundaries of the massive $1+1$ dimensional Nambu-Jona-Lasinio model with isospin, Phys. Rev. D 101, 074013 (2020).

[57] L. Pannullo, J. Lenz, M. Wagner, B. Wellegehausen, and A. Wipf, Inhomogeneous phases in the $1+1$ dimensional Gross-Neveu model at finite number of fermion flavors, Acta Phys. Pol. B Proc. Suppl. 13, 127 (2020).

[58] L. Pannullo, J. Lenz, M. Wagner, B. Wellegehausen, and A. Wipf, Lattice investigation of the phase diagram of the $1+1$ dimensional Gross-Neveu model at finite number of fermion flavors, Proc. Sci., LATTICE2019 (2019) 063 [arXiv:1909.11513].

[59] J. Lenz, L. Pannullo, M. Wagner, B. Wellegehausen, and A. Wipf, Inhomogeneous phases in the Gross-Neveu model in $1+1$ dimensions at finite number of flavors, Phys. Rev. D 101, 094512 (2020).

[60] R. Narayanan, Phase diagram of the large $N$ Gross-Neveu model in a finite periodic box, Phys. Rev. D 101, 096001 (2020).

[61] S. A. Brazovskii, Phase transition of an isotropic system to a nonuniform state, Zh. Eksp. Teor. Fiz. 68, 175 (1975) [Sov. Phys. JETP 41, 85 (1975)].

[62] D. D. Ling, B. Friman, and G. Grinstein, First- and secondorder transitions in models with a continuous set of energy minima, Phys. Rev. B 24, 2718 (1981).

[63] A. M. Dyugaev, Crystalline and liquid phases of a pion condensate, Pis'ma Zh. Eksp. Teor. Fiz. 35, 341 (1982) [JETP Lett. 35, 420 (1982)].

[64] P. C. Hohenberg and J. B. Swift, Metastability in fluctuation-driven first-order transitions: Nucleation of lamellar phases, Phys. Rev. E 52, 1828 (1995).

[65] S. Karasawa, T.-G. Lee, and T. Tatsumi, BrazovskiiDyugaev effect on the inhomogeneous chiral transition 
in quark matter, Prog. Theor. Exp. Phys. 2016, 043D02 (2016).

[66] A. Erzan and G. Stell, Isotropic Lifshitz point in $2<d<4$ dimensions, Phys. Rev. B 16, 4146 (1977).

[67] J. Sak and G. S. Grest, Critical exponents for the Lifshitz point: Epsilon expansion, Phys. Rev. B 17, 3602 (1978).

[68] G. S. Grest and J. Sak, Low-temperature renormalization group for the Lifshitz point, Phys. Rev. B 17, 3607 (1978).

[69] A. Bonanno and D. Zappala, Isotropic Lifshitz critical behavior from the functional renormalization group, Nucl. Phys. B893, 501 (2015).

[70] D. Zappala, Isotropic Lifshitz point in the $\mathrm{O}(\mathrm{N})$ theory, Phys. Lett. B 773, 213 (2017).

[71] D. Zappala, Indications of isotropic Lifshitz points in four dimensions, Phys. Rev. D 98, 085005 (2018).

[72] A. M. Tsvelik, Quantum Field Theory in Condensed Matter Physics (Cambridge University Press, Cambridge, England, 2005).

[73] M. Moshe and J. Zinn-Justin, Quantum field theory in the large N limit: A review, Phys. Rep. 385, 69 (2003).

[74] In a spatially inhomogeneous condensate along the $z$ direction, the inverse propagator for the longitudinal mode is anistropic, $\sim\left(k_{z}-k_{0}\right)^{2}+\cdots$, Eq. (72) [1-3,11-15, $31,32,61-65,75-77]$. A term linear in $k_{z}, \sim-2 k_{0} k_{z}$ is allowed because the condensate spontaneously breaks the rotational symmetry. Instead, Eq. (5) of Ref. [10] introduces a term $\sim-k_{0} \sqrt{\boldsymbol{k}^{2}}$. This does not involve the direction of the condensate and so, unlike ordinary effective Lagrangians, is not analytic in $\boldsymbol{k}^{2}$. This term generates a double pole at nonzero momentum for both the longitudinal and transverse modes and so disorders for all $N$; we only find disorder for $N>2$. Similar to our analysis, Ref. [10] finds that the double pole is absent at zero temperature, as with our Eq. (74); the model is solved at large $N$, similar to our Sec. IV, although since the model differs, so do the details. More generally, we argue for a double pole to arise from an effective Lagrangian which has a well defined derivative expansion, then it must involve higher spatial derivatives.

[75] M. Nitta, S. Sasaki, and R. Yokokura, Spatially modulated vacua in relativistic field theories, Eur. Phys. J. C 78, 754 (2018).

[76] M. Nitta, S. Sasaki, and R. Yokokura, Supersymmetry breaking in spatially modulated vacua, Phys. Rev. D 96, 105022 (2017).

[77] S. B. Gudnason, M. Nitta, S. Sasaki, and R. Yokokura, Temporally, spatially, or lightlike modulated vacua in Lorentz invariant theories, Phys. Rev. D 99, 045011 (2019).

[78] Reference [32] considers fluctuations which multiply the background field, $\boldsymbol{\phi}=\left(\boldsymbol{\phi}_{0}+\sigma_{q}\right) \exp \left(i t^{a} \phi_{q}^{a}(x)\right)$, where $t^{q}$ are generators of $O(4)$. The first term on the right-hand side of their Eq. (15) does exhibit a double pole in terms of their $\boldsymbol{\beta}_{U}=\boldsymbol{\beta}_{T} \cos \left(k_{0} z\right)$, but the spectrum of their $\boldsymbol{\beta}_{T}$ does not appear to exhibit a double pole, Eqs. (16)-(20) and Appendix B of Ref. [32]. We expand in linear fluctuations about the background field, $\boldsymbol{\phi}=\left(\boldsymbol{\phi}_{0}+\boldsymbol{\sigma}_{q}, \chi\right)$, our Eq. (31), where $\chi$ is related to their $\boldsymbol{\beta}_{U}$ and $\boldsymbol{\beta}_{T}$. There is a double pole at nonzero momentum in the static inverse propagator for $\chi$, Eqs. (14), (15), (22), and (43).

[79] Reference [31] considers a kink crystal in a NambuJona-Lasino (NJL) model in $3+1$ dimensions, and find anisotropic Goldstone modes about zero momentum. As discussed in Sec. VI A, the NJL model only breaks a global $Z(2)$ symmetry. As we discuss in Sec. II B, we expect that the spontaneous breaking of an $\mathrm{O}(4)$ symmetry prefers a chiral spiral over a kink crystal.

[80] L. B. Ioffe and A. I. Larkin, Effective action of a twodimensional antiferromagnet, Int. J. Mod. Phys. B 02, 203 (1988).

[81] Y. Zhou, K. Kanoda, and Tai-Kai Ng, Quantum spin liquid states, Rev. Mod. Phys. 89, 025003 (2017).

[82] L. Savary and L. Balents, Quantum spin liquids: A review, Rep. Prog. Phys. 80, 016502 (2017).

[83] Y. A. Kharkov, O. P. Sushkov, and M. Mostovoy, Bound States of Skyrmions and Merons Near the Lifshitz Point, Phys. Rev. Lett. 119, 207201 (2017).

[84] Y. A. Kharkov, J. Oitmaa, and O. P. Sushkov, Properties of the spin-liquid phase in the vicinity of the Lifshitz transition from Néel to spin-spiral state in frustrated magnets, Phys. Rev. B 98, 144420 (2018).

[85] Y. A. Kharkov, J. Oitmaa, and O. P. Sushkov, Quantum Lifshitz criticality in a frustrated two-dimensional $\mathrm{XY}$ model, Phys. Rev. B 101, 035114 (2020).

[86] M. C. O'Brien and O. P. Sushkov, Colossal quasiparticle radiation in the Lifshitz spin liquid phase of a twodimensional quantum antiferromagnet, Phys. Rev. B 101, 184408 (2020).

[87] M. Asakawa and K. Yazaki, Chiral restoration at finite density and temperature, Nucl. Phys. A504, 668 (1989).

[88] M. A. Stephanov, K. Rajagopal, and E. V. Shuryak, Signatures of the Tricritical Point in QCD, Phys. Rev. Lett. 81, 4816 (1998).

[89] M. A. Stephanov, K. Rajagopal, and E. V. Shuryak, Eventby-event fluctuations in heavy ion collisions and the QCD critical point, Phys. Rev. D 60, 114028 (1999).

[90] D. T. Son and M. A. Stephanov, Dynamic universality class of the QCD critical point, Phys. Rev. D 70, 056001 (2004).

[91] M. A. Stephanov, Non-Gaussian Fluctuations Near the QCD Critical Point, Phys. Rev. Lett. 102, 032301 (2009).

[92] P. Parotto, M. Bluhm, D. Mroczek, M. Nahrgang, J. Noronha-Hostler, K. Rajagopal, C. Ratti, T. Schäfer, and M. Stephanov, Lattice-QCD-based equation of state with a critical point, Phys. Rev. C 101, 034901 (2020).

[93] B.-J. Schaefer and J. Wambach, Susceptibilities near the QCD (tri)critical point, Phys. Rev. D 75, 085015 (2007).

[94] F. Rennecke and B.-J. Schaefer, Fluctuation-induced modifications of the phase structure in $(2+1)$-flavor QCD, Phys. Rev. D 96, 016009 (2017).

[95] W.-j. Fu, J. M. Pawlowski, and F. Rennecke, QCD phase structure at finite temperature and density, Phys. Rev. D 101, 054032 (2020).

[96] A. Bzdak, S. Esumi, V. Koch, J. Liao, M. Stephanov, and $\mathrm{N}$. Xu, Mapping the phases of quantum chromodynamics with beam energy scan, Phys. Rep. 853, 1 (2020).

[97] S. Valgushev (to be published). 
[98] D. J. Gross and A. Neveu, Dynamical symmetry breaking in asymptotically free field theories, Phys. Rev. D 10, 3235 (1974).

[99] R. F. Dashen, B. Hasslacher, and A. Neveu, Semiclassical bound states in an asymptotically free theory, Phys. Rev. D 12, 2443 (1975).
[100] R. D. Pisarski, Chiral symmetry breaking in threedimensional electrodynamics, Phys. Rev. D 29, 2423 (1984).

[101] R. D. Pisarski, F. Rennecke, A. Tsvelik, and S. Valgushev, The Lifshitz regime and its experimental signals, arXiv: 2005.00045 [Nucl. Phys. A (to be published)]. 